\title{
Longitudinal Strain Induced Birefringence in Highly Birefringent Fiber for Dynamic Compensation of Polarization Mode Dispersion
}

\author{
A.W. Domański*, P. Lesiak and T.R. Woliński \\ Faculty of Physics, Warsaw University of Technology \\ Koszykowa 75, 00-662 Warsaw, Poland
}

\begin{abstract}
The paper addresses a compensation method of polarization mode dispersion based on longitudinal strain applied to a highly birefringent fiber introduced as apart from optical fiber link. As an introduction a short overview of the polarization mode dispersion compensation methods known up-to-now is done. Dependence of polarization mode dispersion on longitudinal strain is discussed. Some results of experiment with birefringence changes due to longitudinal strain in the bow-tie highly birefringent fiber necessary for dynamic polarization mode dispersion are presented.
\end{abstract}

PACS numbers: 42.81.Gs, 42.81.Pa, 42.25.Ja, 42.25.Dd

\section{Introduction}

Polarization mode dispersion (PMD) strongly diminishes bandwidth of transmitted signals in optical fiber transmission systems. It is caused by randomly distributed residual birefringence of any unstressed optical fiber that is commonly used in telecommunication applications. Hence, there are a lot of attempts to compensate for birefringence fluctuation in optical fibers and in the same to compensate for polarization mode dispersion.

The major cause of polarization mode dispersion is the asymmetry of the fiber optic cross-section. Asymmetry is caused by the fact that the fiber core is slightly not round or elliptical. When the core of the fiber is asymmetrical, the light traveling along a birefringence axis moves slower (or faster) than the light polarized along the orthogonal axis. This effect can spread the pulse enough

*corresponding author; e-mail: domanski@if.pw.edu.pl 
to make it overlap with other pulses or change its own shape enough to make it undetectable at the receiver. Fiber asymmetry may be inherent in the fiber from the manufacturing process, or it may be a result of mechanical stress on the deployed fiber. The mechanical stress can originate from a variety of sources. One source that is very difficult to control is diurnal (day/night) and seasonal heating and cooling of the optical fiber. Although many fibers are deployed in the ground and often within conduits, they are still subjected to temperature variations and corresponding mechanical stresses. Another source of mechanical stress can originate from nearby sources of vibration.

Polarization mode dispersion does not have a single value for a given section of fiber. Rather, it is described in terms of average differential group delay (DGD), and a fiber has a distribution of DGD values over time. The probability of the DGD of a fiber section being a certain value at any particular time follows the Maxwell distribution.

In the present paper we have introduced a novel dynamic method of PMD compensation that uses a highly birefringent optical fiber in which changes in DGD are induced by longitudinal strain.

\section{Methods of PMD compensation}

The simplest technique to compensate for polarization mode dispersion is known as the first-order PMD compensation (or the principal states of polarization method) [1], since it only enables compensation of the first-order PMD. This method is realized by introduction of the optical signal into a principle state of polarization at the input of the fiber optic system as shown in Fig. 1a. The polarization control (PC) is made with fast electro-optical crystals.

There are several other methods of PMD compensation realized after propagation of the signals in optical fiber links (Fig. 1b), in which compensation may be achieved by the use of static or dynamic methods.

(a)

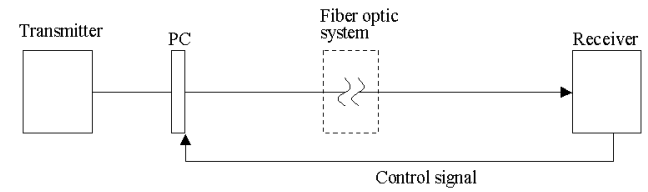

(b)

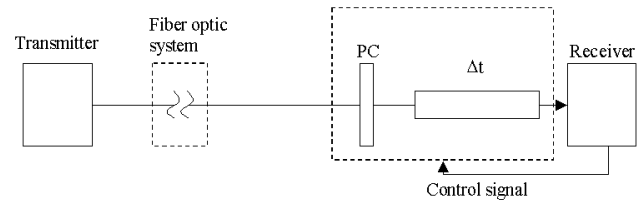

Fig. 1. Methods of PMD compensation; (a) pre-propagate method, (b) after propagate method. 
Static methods require a polarizer or, based on fixed time delay, a properly oriented birefringent fiber [2]. The polarizer shifts one state of polarization enabling an attractive way of reducing pulse broadening. Another static method of PMD compensation is based on the assumption that an optical signal launched into the fiber is characterized by a constant differential group delay [3,4]. The single DGD element can drastically reduce the average pulse spreading, and hence, the average bit error rate. To control input states of polarization a personal computer is commonly used. This system is much more simpler to build, control, and analyze and therefore is superior to the first-order PMD compensation system.

Dynamic methods or variable delay methods are based on application of optical elements with electronically controlled optical pulse to compensate for the DGD [5]. These methods are generally based on variation in the differential group delay. This allows compensating randomly changes in PMD around its average value. There are several physical phenomena that can be used for dynamic compensation of PMD. The compensation of variable delay may be achieved by phase shift caused by electrooptical, elastooptical or magnetooptical methods. Here bulk materials as well as optical fibers may serve as optical active element.

A comparison between different methods of PMD compensation is shown in the Table. The fifth column in the Table shows parameters of the $n$-degree of freedom method that is a combination of several other PMD compensation methods. It allows obtaining additional degrees of freedom in comparison with other PMD compensators. The higher the number of degree of freedom, the system becomes more flexible. Moreover, enabling compensation for various DGD values and to some extent also for higher orders of PMD. On the other hand, any increase in the degree of freedom complicates the whole PMD compensation system. This slows down the response and consequently reduces the probability of maintaining an optimized state of the compensating system.

TABLE

Comparison between different PMD compensation techniques.

\begin{tabular}{c|c|c|c|c|c}
\hline \hline & $\begin{array}{c}\text { First } \\
\text { order }\end{array}$ & Polarizer & $\begin{array}{c}\text { Fixed time } \\
\text { delay }\end{array}$ & $\begin{array}{c}\text { Variable } \\
\text { delay }\end{array}$ & $\begin{array}{c}n \text {-degree of } \\
\text { freedom }\end{array}$ \\
\hline $\begin{array}{c}\text { System } \\
\text { complexity }\end{array}$ & low & low & low & average & high \\
\hline $\begin{array}{c}\text { Response } \\
\text { speed }\end{array}$ & fast & fast & fast & medium & slow \\
\hline $\begin{array}{c}\text { Quality of } \\
\text { compensation }\end{array}$ & low & average & average & high & high \\
\hline $\begin{array}{c}\text { Order of PMD } \\
\text { compensation }\end{array}$ & $\begin{array}{c}\text { first } \\
\text { order }\end{array}$ & $\begin{array}{c}\text { first } \\
\text { order }\end{array}$ & $\begin{array}{c}\text { first } \\
\text { order }\end{array}$ & $n$-order & $n$-order
\end{tabular}




\section{Dependence of differential group delay on longitudinal strain}

The dynamic method of PMD compensation utilizing a highly birefringent (HB) fiber is based on mechanically induced changes in differential group delay in the short section of the HB fiber.

Differential group delay $\Delta \tau$ in the HB fiber strongly depends on modal birefringence $\Delta \beta$ of the fiber as follows:

$$
\frac{\Delta \tau}{L}=\frac{\mathrm{d}(\Delta \beta)}{\mathrm{d} \omega}=\frac{1}{c}\left(\Delta n_{\mathrm{eff}}+\omega \frac{\mathrm{d} \Delta n_{\mathrm{eff}}}{\mathrm{d} \omega}\right)\left[\frac{\mathrm{ps}}{\mathrm{km}}\right],
$$

where $\Delta \tau / L$ is usually expressed in units of picoseconds per kilometer of fiber length, $\Delta n_{\mathrm{eff}}$ is the differential effective index of refraction for the slow and fast polarization modes, and $\omega=2 \pi c / \lambda$ is the angular frequency of light.

For HB fibers in which birefringence is caused by stress applying parts introduced in cladding close to the core region of the fiber $\Delta n_{\text {eff }}$ is nearly wavelength independent and the chromatic dispersion of the modal birefringence is negligible. Hence for this type of fiber the measurements of birefringence and PMD are equivalent and the formula (1) may by reduced to the following expression:

$$
\frac{\mathrm{d}(\Delta \beta)}{\mathrm{d} \omega} \cong \frac{1}{c} \Delta n_{\mathrm{eff}}=\frac{\Delta \beta}{c k}
$$

Typical values of DGD for HB fibers are close to $1000 \mathrm{ps} / \mathrm{km}$, that is relatively very high in comparison to $0.1 \mathrm{ps} / \mathrm{km}$ (typical values of PMD for telecommunication fibers). Hence, we propose to use a short section of the HB fiber to compensate changes in DGD and consequently in PMD of telecommunication fibers.

Controlled variations of birefringence in HB fibers necessary for compensation of random fluctuations of birefringence in telecommunication fibers may be caused by such external parameters as axial and radial stresses, hydrostatic pressure or longitudinal strain. The effect of externally induced birefringence as this observed in HB bow-tie fibers due to different above-mentioned environmental influences is well known and has been investigated in details elsewhere [6]. For example, the influence of longitudinal strain can be expressed by the following formula:

$$
\Delta \beta=\Delta \beta_{0}+2 \pi \varepsilon /\left(T_{\varepsilon} L\right),
$$

where $\varepsilon$ is the longitudinal strain defined as $\Delta L / L, L$ is the length of the fiber, and $T_{\varepsilon}$ is an experimental parameter corresponding to the amount of strain required to induce a $2 \pi$ phase shift of the polarized light observed at the output [7]. Hence, differential group delay for a highly birefringent fiber in which birefringence is caused by stress applying parts depends on longitudinal strain as follows:

$$
\frac{\Delta \tau}{L}=\frac{\Delta \beta}{c k}=\frac{\Delta \beta_{0}}{c k}+\frac{2 \pi \varepsilon}{T_{\varepsilon} L c k} \text {. }
$$

Since the product of $T_{\varepsilon}$ and $L$ is constant and equal to the value that depends only on the wavelength used and the type of the HB fiber, hence the total birefringence 
$\Delta \beta$ increases with strain. Due to the fact that longitudinal strain in the fiber can be easily changed, for example by using a piezoelectric cylinder with the HB fiber wounded on it we can compensate in a simple way DGD of the incoming optical signals caused by random fluctuation of birefringence in a telecommunication fiber.

\section{Experimental results}

The differential group delay induces not only polarization mode dispersion but also diminishes the degree of polarization (DOP) of the light passing through the highly birefringent fibers [8,9]. When the DGD of the output light increases, then the DOP decreases in different way depending on coherence of the light source used in experiments. Strict theoretical relationship between DGD, DOP, and coherence of light is unknown but measurements of changes in DOP are helpful for prediction of longitudinal strain influence on PMD compensation. Hence, instead of direct measurement of the DGD, caused by longitudinal strain, we introduce an indirect method based on strain-induced changes in the DOP of the light coming out of the HB fiber in order to check a possibility of dynamic compensation of PMD by the use of the method.

In our laboratory, we have constructed a system to measure the state and the degree of polarization of the output light. This system is called the polarization analyzing system, version 1 (PAS-1). The system can measure four light intensities, for four different arrangements of the analyzer and the quarter wave plate and then automatically calculates four Stokes parameters along with intensity of the output light as well as its DOP parameter [10].

In our system (Fig. 2), a laser diode operating at $670 \mathrm{~nm}$ wavelength is used as a light source. The linearly polarized light is launched into the bow-tie HB 600 fiber and then is analyzed by the PAS-1 polarimeter. The polarimeter was also connected to a PC computer, which calculated the DOP parameter. The HB fiber was fixed and exposed to the longitudinal strain.

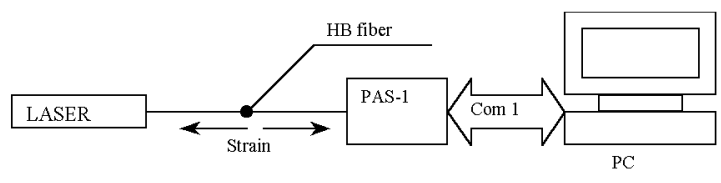

Fig. 2. Experimental setup for a degree of polarization measurement.

When the linearly polarized light at an angle $0^{\circ}$ with respect to birefringence axes was injected to the HB fiber, the degree of polarization of the output light was near its maximal value (equal to 1 ). However, if the linearly polarized light at an angle $45^{\circ}$ with respect to birefringence axes was injected to the HB fiber, the degree of polarization (Fig. 3) of the output light depended on the length of 


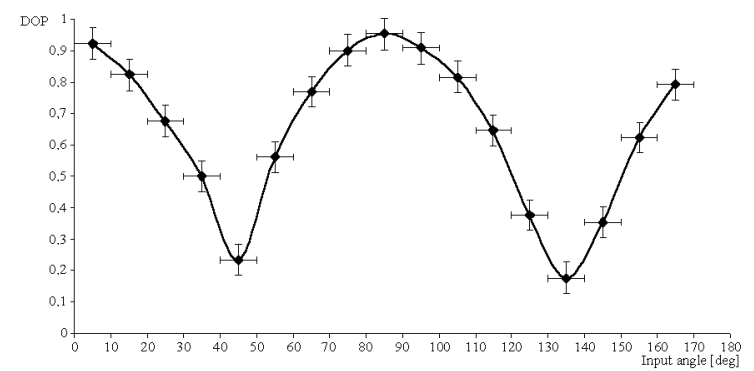

Fig. 3. DOP as a function of the input angle of the linearly polarized light for the HB bow-tie fiber (1.5 $\mathrm{m}$ in length).

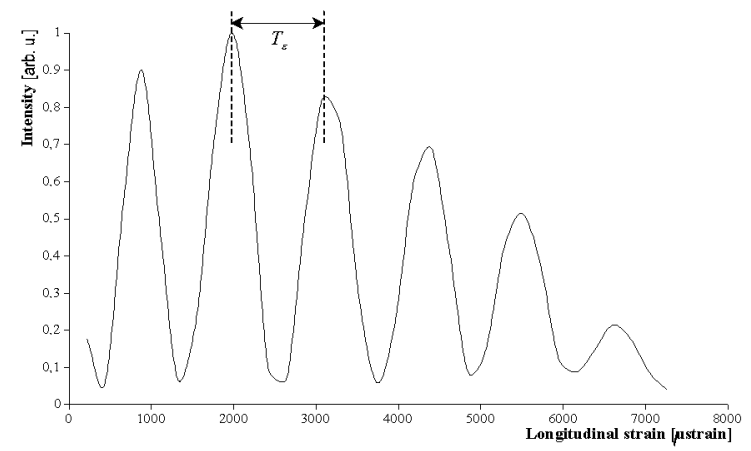

Fig. 4. Intensity of the output light as a function of longitudinal strain for HB bow-tie fiber.

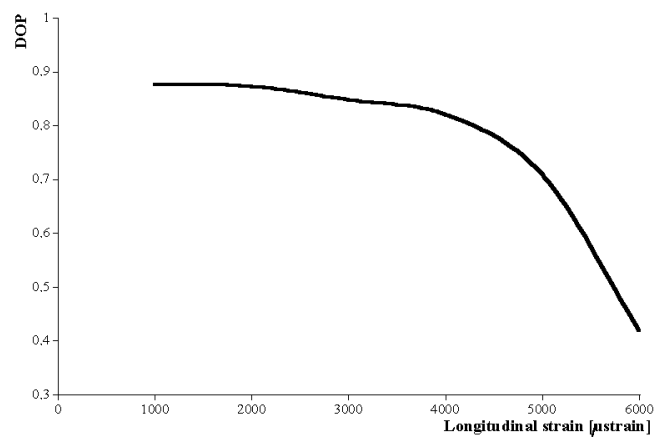

Fig. 5. DOP as a function of longitudinal strain for HB bow-tie fiber.

the fiber, its birefringence as well as the coherence of light source. Intensity of the output light is shown in Fig. 4. The observed decreasing dynamics of the output characteristics of the HB fiber subjected to the longitudinal strain can be directly attributed to its polarization mode dispersion. As a consequence, the degree of polarization of the output light also diminishes as presented in Fig. 5. 


\section{Conclusions}

We have introduced a novel method to compensate for polarization mode dispersion in telecommunication optical fibers based on dynamically longitudinal strain-induced changes in birefringence of highly birefringent bow-tie fibers. This variable delay method offers certain advantages as reduction of the average pulse spreading as well as bit error rate and simultaneously allows compensating randomly changes in PMD around its average value.

\section{Acknowledgment}

The work was supported by the State Committee for Scientific Research (KBN) under the grant 8T11D027019.

\section{References}

[1] H. Sunnerud, C. Xie, M. Karlsson, R. Samuelsson, P.A. Andrekson, J. Lightwave Technol. 20, 368 (2002).

[2] R. Noé, D. Sandel, M. Yoshida-Dierolf, S. Hinz, V. Miroda, A. Schöpflin, C. Glingener, E. Gottwald, C. Scheerer, G. Fischer, T. Weyrauch, W. Haase, J. Lightwave Technol. 17, 1602 (1999).

[3] T. Takahashi, T. Imai, M. Aiki, Electron. Lett. 30, 348 (1994).

[4] I.T. Lima, Jr., G. Biondini, B.S. Marks, W.L. Kath, C.R. Menyuk, IEEE Photon. Technol. Lett. 14, 627 (2002).

[5] C. Francia, F. Bruyère, J.P. Thiéry, D. Penninckx, Electron. Lett. 35, 414 (1999).

[6] T.R. Wolinski, in: Progress in Optics, Ed. E. Wolf, Vol. XL, North Holland, Amsterdam 2000, p. 1.

[7] W.J. Bock, T.R. Woliński, T. Eftimov, Pure Appl. Opt., J. Europ. Opt. Soc. 5, 125 (1996).

[8] H. Rosenfeldt, R. Ulrich, E. Brinkmeyer, U. Feiste, C. Schubert, J. Berger, R. Ludwig, M.G. Weber, A. Ehrhardt, in: ECOC'2001, Amsterdam (Netherlands) 2001, p. 68 .

[9] N. Kikuchi, J. Lightwave Technol. 19, 480 (2001).

[10] Ch. Brosseau, Fundamentals of Polarized Light: a Statistical Optics Approach, John Wiley \& Sons Inc., New York 1998. 